\title{
Surgical Repair for Anomalous Origin of the Right Coronary Artery from the Pulmonary Artery
}

\author{
Alwaleed Al-Dairy, MD¹, Yousef Rezaei, MD², Hamidreza Pouraliakbar, MD³, Mohammad Mahdavi, MD4, \\ Parvin Bayati, $\mathrm{MN}^{4}$, and Maziar Gholampour-Dehaki, MD \\ ${ }^{1}$ Division of Congenital Cardiac Surgery, Department of Cardiovascular Surgery, ${ }^{2}$ Heart Valve Disease Research Center, ${ }^{3}$ Department of Radiology, ${ }^{4}$ Department of Pediatric \\ Cardiology, Rajaie Cardiovascular Medical and Research Center, Iran University of Medical Sciences, Tehran, Iran
}

Anomalous origin of the right coronary artery from the pulmonary artery (ARCAPA) is a very rare congenital heart defect. Herein, we describe three cases of ARCAPA in an 8 months old, 18 months old, and 4 year old child. Two cases were incidentally diagnosed using a computed tomographic angiograph, and the other was incidentally diagnosed using a coronary angiograph. These cases underwent a reimplantation technique on diagnosis and resulting in positive clinical outcomes during the follow-up period which was a mean of 1.5 years. (Korean Circ J 2017;47(1):144-147)

KEY WORDS: Congenital abnormalities; Coronary vessel anomalies; Cardiac surgical procedures; Electrocardiography.

\section{Introduction}

Anomalous origin of the right coronary artery from the pulmonary artery (ARCAPA) is a very rare congenital heart defect. ${ }^{1-3)}$ Most ARCAPA cases are isolated anomalies, but approximately $25-30 \%$ is found to be associated with other structural heart defects. ${ }^{4}$ In 1885 , the first cases of ARCAPA were described by Brooks. ${ }^{5}$ Most affected patients are asymptomatic and their anomalies were detected incidentally. This is completely different from the anomalous origin of the left coronary artery from the pulmonary artery (ALCAPA), which without definitive surgical treatment can frequently lead to death early in life. ${ }^{6)}$ However, sudden cardiac death from ARCAPA

Received: May 3, 2016

Revision Received: May 30, 2016

Accepted: May 31, 2016

Correspondence: Maziar Gholampour-Dehaki, MD, Division of

Congenital Cardiac Surgery, Department of Cardiovascular Surgery, Rajaie Cardiovascular Medical and Research Center, Tehran 1996911151, Iran

Tel: 98-2122040727, Fax: 98-2122040727

E-mail:drgholampour@yahoo.com

- The authors have no financial conflicts of interest.

This is an Open Access article distributed under the terms of the Creative Commons Attribution Non-Commercial License (http://creativecommons. org/licenses/by-nc/3.0) which permits unrestricted non-commercial use, distribution, and reproduction in any medium, provided the original work is properly cited. has been reported as well.7) The most common symptoms which would require the patient to seek medical attention are murmurs followed by chest pain, and symptoms of congestive heart failure.? The natural history of ARCAPA is difficult to describe because of the relatively small number of reported cases. Definitive therapy requires surgical translocation of the anomalous right coronary artery (RCA) to the aortic root. ${ }^{6}$

\section{Case}

Patient no. 1 was an 8-month-old male with a weight of $7.5 \mathrm{~kg}$ suffering from seizures. Electrocardiographic (ECG) examination revealed an inverted $\mathrm{T}$ wave in leads $\mathrm{V}_{1}-\mathrm{V}_{4}$. On transthoracic echocardiogram (TTE), there was a dilated left main coronary artery $(0.24 \mathrm{~cm}$ diameter-identified according to online software, version 3.7.0 [Ped(z)-Pediatric Calculator; Daniel Gräfe, Germany] for calculating coronary artery Z score) with a suspected diagnosis of ARCAPA associated with good left ventricular ejection fraction (LVEF). A computed tomographic angiography (CTA) was carried out and confirmed the diagnosis of ARCAPA and also revealed an aberrant right subclavian artery along with a right dominant coronary circulation. The patient underwent reimplantation of the anomalous RCA (which was originating from the right anterolateral aspect of the pulmonary artery [PA]) into the ascending aorta. For 
that, a complete cardiopulmonary bypass (CPB) with aortobicaval cannulation was prepared and moderate hypothermia $\left(28-32^{\circ} \mathrm{C}\right)$ was used. Then, antegrade cold blood cardioplegia was administered with the pulmonary arteries snared. A large coronary ostial button, which contains the RCA, was harvested from the pulmonary artery, and the RCA was mobilized as well. Then, an opening was made in the left anterolateral aspect of the aorta followed by direct implantation of the RCA. The defect in the sinus of the pulmonary artery was reconstructed with a fresh autologous pericardial patch. The patient was weaned off the CPB without inotrope support. In the intensive care unit (ICU) a low dose of Milrinone $(0.25 \mathrm{mcg} / \mathrm{kg} / \mathrm{min})$ was administered along with unfractioned Heparin infusion with a dose of 50 units/hour in order to keep the partial thromboplastin time (PTT) between 60 to 90 seconds. The intubation time lasted
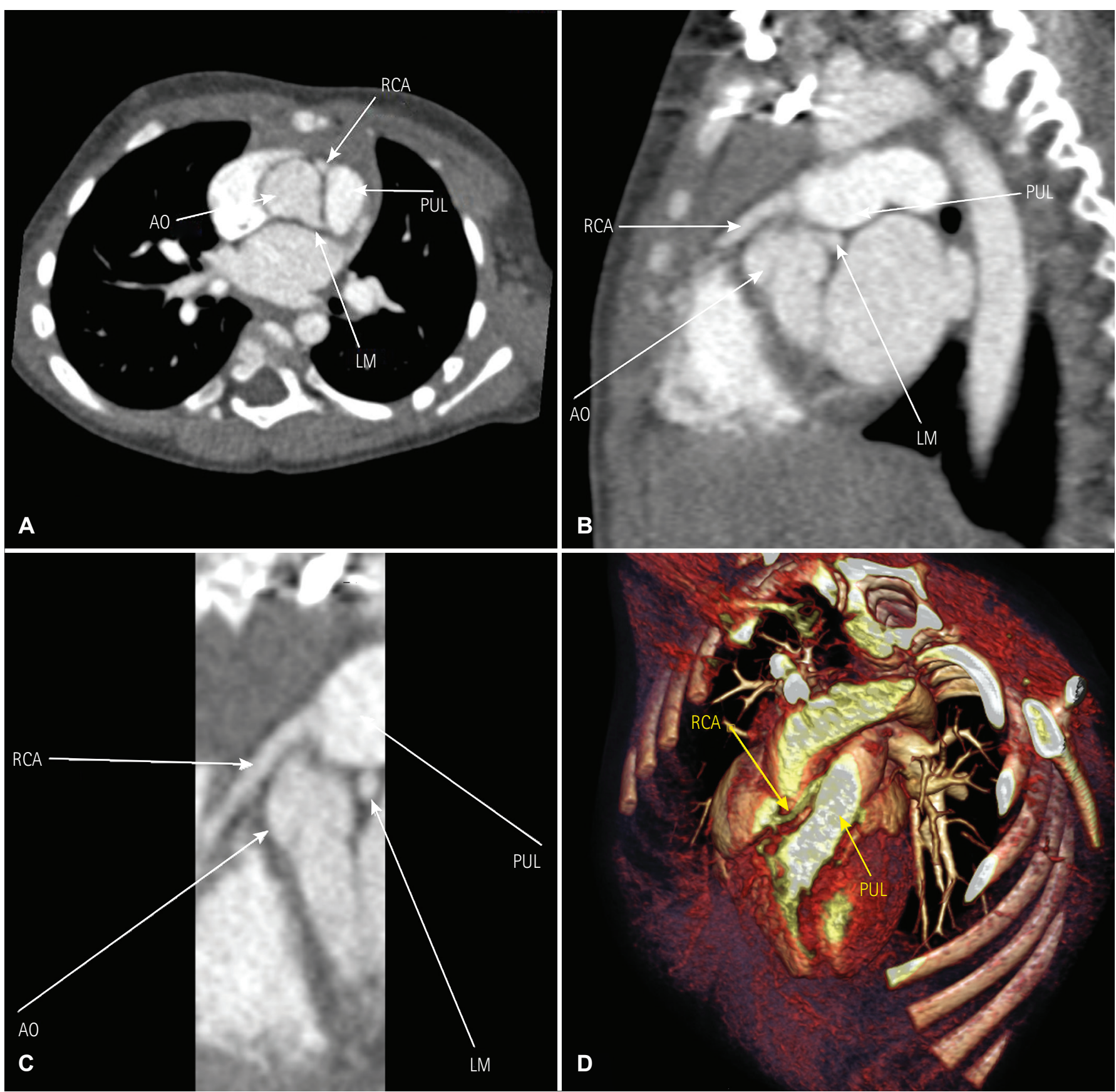

Fig. 1. Preoperative diagnostic images of the anomalous right coronary artery. (A) Computed tomographic angiography showing the anomalous right coronary artery originating from the pulmonary artery in coronal view, (B) sagittal view, (C) zoomed view, and (D) three-dimensional view. RCA: right coronary artery, AO: aorta, PUL: pulmonary artery, LM: left main coronary artery. 
16 hours and the patient was discharged after 5 days on digoxin, captopril, aspirin, phenobarbital, and propranolol. At the 3-year follow-up period, the patient appeared well, and was without any dyspnea or chest pain along with a LVEF of $65 \%$ measured by TTE.

Patient no.2 was an 18-month-old male with a weight of $9.8 \mathrm{~kg}$ who was asymptomatic; during a routine checkup a 2/6 systolic murmur was heard in the left second intercostal space, ECG was normal. However, TTE demonstrated a dilated left coronary artery (0.3 cm diameter) and normal left and right ventricular function without any associated lesions. CTA revealed an RCA originated from the right side of the PA with an anterior course in relation to the ascending aorta along with a right dominant coronary system (Fig. 1). The patient underwent reimplantation of the anomalous RCA into the ascending aorta, with the origin of the RCA from the right facing sinus of the PA adjacent to the commissure. The patient was weaned off the CPB without inotrope support. In the ICU Milrinone with a dose of $0.5 \mathrm{mcg} / \mathrm{kg} / \mathrm{min}$ was administered along with unfractioned Heparin infusion with a dose of 100 units/hour. The intubation time lasted 26 hours and the patient was discharged after 6 days on aspirin and furosemide. At the one-year follow-up, the patient was asymptomatic (no chest discomfort or dyspnea) with a LVEF of $66 \%$ measured by TTE.

Patient no. 3 was a 4-year-old male with a weight of $15 \mathrm{~kg}$ who was asymptomatic. However, during hospitalization for upper respiratory tract infection, the ECG revealed an inverted $T$ wave in leads $V_{1}-V_{4}$ and consecutively TTE was performed and demonstrated a dilated left coronary artery with normal left and right ventricular functions. Coronary angiography demonstrated a dilated left main coronary artery and a retrograde flow in the RCA with runoff into the PA with a left dominant coronary circulation. The patient underwent reimplantation of the anomalous RCA into the ascending aorta, with the origin of the RCA from the right facing sinus of the PA. Postoperative management was identical to previous cases. The intubation time lasted 40 hours during which he was extubated for the first time after 16 hours but reintubated because of agitation and restlessness. He was then discharged after 6 days on digoxin, captopril, aspirin, and furosemide. The patient did not show any signs of dyspnea or chest pain and had a LVEF of 70\% measured by TTE at 6-month follow-up.

\section{Discussion}

Coronary artery anomalies arise embryologically as a result of either malrotation of the spiral septum dividing the truncus or malpositioning of the coronary buds themselves. The higher incidence of ALCAPA compared to ARCAPA may be explained by the proximity of the left coronary bud to the PA sinus. The pathophysiology of ARCAPA depends on the direction of blood flow in the coronary artery and its influence on oxygen delivery to the myocardium. ${ }^{8)}$ The severity of ventricular ischemia is determined by the shunt size, the degree of collateral circulation, and myocardial oxygen demands.7) Because right ventricular oxygen demands are lower than that of the left ventricle, the ventricular ischemia in the ARCAPA is less prominent than in the ALCAPA.?) However, patients with right dominant coronary circulation do not tolerate ARCAPA compared with those with a left dominant system. ${ }^{8}$ In our series, the 8 and 18-month-old patients had right dominant coronary circulations, while the 4-year-old patient had a left dominant one. Although all patients presented asymptomatically with preserved left and right ventricular function, the role of coronary circulation dominance on ARCAPA presentation cannot be confirmed.

The diagnosis of ARCAPA is mainly incidental, ${ }^{6}$ but some patients present with a murmur, chest pain, or symptoms of congestive heart failure.7) However, the patients presented in this report did not suffer from cardiac symptoms, and only one patient had a murmur, which led to the diagnosis of ARCAPA. On the other hand, two other patients had ischemic changes on an ECG found in the setting of routine check-up for non-cardiac complaints. Moreover, ventricular function was also preserved in these cases.

Advances in diagnostic technologies have resulted in an increase in the rate of diagnosis of ARCAPA during infancy and early childhood. ${ }^{8)}$ Cardiac CTA and cardiac magnetic resonance is a gold standard diagnostic tool providing an excellent visualization of anomalous coronary arteries." In our cases, the diagnosis was suspected by the TTE as a dilated left coronary artery was detected and confirmed by a CTA in two patients and by angiography in the other. Associated anomalies such as aortopulmonary window, tetralogy of Fallot, and septal defects have been reported in 22\% of patients. ${ }^{6)}$ However, except for an aberrant right subclavian artery in one patient, the ARCAPA was an isolated anomaly in our patients.

Surgery is indicated on the diagnosis of ARCAPA, even in asymptomatic patients to prevent the development of myocardial ischemia and sudden cardiac death. ${ }^{9}$ Different surgical procedures have been described for the management of ARCAPA. The ligation of RCA at its origin from the pulmonary trunk with or without concomitant grafting to the RCA is one option. However, the long-term patency of the graft is debatable. . $^{9}$ Another option is the reimplantation of the anomalous RCA from the pulmonary artery into the anterior wall of the ascending aorta to reestablish a dual coronary artery system, which results in a better outcome compared with single coronary ostium system. ${ }^{910)}$ In our cases, we used the reimplantation technique in all patients, which was also associated with no cases of in-hospital mortality. 
Reports describing follow-up of ARCAPA after surgical correction are rare. ${ }^{8)}$ The follow-up period of our cases was six months, one year, and three years, and neither late mortality nor complications were detected during the follow-up period. However, the risk for potential late complications exists, and long-term follow-up of patients is warranted. It may be recommended to perform an annual postoperative echocardiographic screening due to some studies reporting an incidence of clot in the reimplanted RCA.

In conclusion, the use of reimplantation techniques for ARCAPA repair can result in good outcomes with the establishment of a dual coronary system to prevent the continuous risk of sudden cardiac death and myocardial ischemia in such cases.

\section{References}

1. Li RJ, Yang Y, Li ZA, Xie JJ, Zhang XS. Right coronary artery arising from the main pulmonary artery: Evaluation with 2-dimensional transthoracic echocardiography and multislice computed tomography. Tex Heart Inst J 2010;37:376-7.

2. Esther C, Park JJ, Yoon TJ, Kim YH, Ko JK, Park IS, et al. Anomalous origin of the right coronary artery from the pulmonary artery. Korean J Thorac Cardiovasc Surg 2002;35:894-7

3. Park CS, Kim W, Hong SB, Hwang SH, Kim W, An BH. Anomalous origin of the right coronary artery from the main pulmonary artery presenting as angina pectoris in an adult. Korean Circ J 2008;38:627-9.

4. Gupta R, Marwah A, Shrivastva S. Anomalous origin of right coronary artery from pulmonary artery. Ann Pediatr Cardiol 2012;5:95-6.

5. Brooks HS. Two cases of an abnormal coronary artery of the heart arising from the pulmonary artery: With some remarks upon the effect of this anomaly in producing cirsoid dilatation of the vessels. $J$ Anat Physiol 1885;20(Pt 1):26-9.

6. Maroules CD, Adams DZ, Whiting ED, Antevil JL, Mitchell ES. Anomalous origin of the right coronary artery from the pulmonary artery: Evaluation with use of 64-slice multidetector computed tomography. Tex Heart Inst J 2013;40:106-8.

7. Winner MW 3rd, Raman SV, Sun BC, Crestanello JA. Preoperative assessment of anomalous right coronary artery arising from the main pulmonary artery. Case Rep Med 2011;2011:642126.

8. Williams IA, Gersony WM, Hellenbrand WE. Anomalous right coronary artery arising from the pulmonary artery: A report of 7 cases and a review of the literature. Am Heart J 2006;152:1004.e9-17.

9. Kuba PK, Sharma J, Sharma A. Successful surgical treatment of a septuagenarian with anomalous right coronary artery from the pulmonary artery with an eleven year follow-up: Case report and review of literature. Sultan Qaboos Univ Med J 2013;13:169-74.

10. Hauser M. Congenital anomalies of the coronary arteries. Heart 2005;91:1240-5. 